\title{
ON THE ISOMORPHISMS OF TWO METACYCLIC GROUPS
}

\author{
B. G. BASMAJI
}

1. Introduction. In [2] Szekeres determined all the metabelian groups of two generators and raised the question of whether a rule of selection exists to determine all the nonisomorphic ones. In this paper we find such a rule for the finite metacyclic groups. That is, we give a rule to determine all the nonisomorphic extensions of a cyclic group of order $n$ by a cyclic group of order $h$.

Our approach uses strongly the defining relations of two extensions. In $\$ 2$ we give the notations, the isomorphism theorem, and the rule that gives all the nonisomorphic groups. In \$3 some preliminary lemmas are proved. The theorem for the case of metacyclic $p$-groups is proved in $\$ 4$ and a remark is made that reduces the proof to a special pair of two metacyclic groups. The necessity and the sufficiency of the conditions are proved in $\$ 5$ and $\$ 6$ respectively. In particular we note in $\$ 6$ that the isomorphism need not be of the first kind as defined by Gol'fand [1].

2. Notations and main result. Let $G$ be an extension of a cyclic group of order $n$ by a cyclic group of order $h$, i.e. $G$ is a metacyclic group. Then the defining relations of $G=\{a, b\}$ are given by

$$
a^{n}=b^{h \theta}=1, \quad a^{k}=b^{h}, \quad b a=a^{r} b,
$$

where $g \mid n, k=n / g, r^{h} \equiv 1(\bmod n)$, and $g \mid r-1$. [Note that there is no loss of generality in assuming that $k \mid n$, and hence $k=n / g$. For if $k \nmid n$, then replacing $k$ by $k_{0}=(k, n)$ and $a$ by $a^{x}$, where $x$ is a solution of $x \equiv k / k_{0}\left(\bmod n / k_{0}\right)$ and $x \equiv 1\left(\bmod n^{\prime}\right), n^{\prime}$ the product of distinct primes $p$ such that $p \mid n$ and $p \nmid n / k_{0}$, gives the above relations.]

Let $H=\{c, d\}$ be another extension with the defining relations

$$
c^{n}=d^{h g^{\prime}}=1, \quad c^{k^{\prime}}=d^{h}, \quad d c=c^{\sigma} d
$$

where $g^{\prime} \mid n, k^{\prime}=n / g^{\prime}, \sigma^{h} \equiv 1(\bmod n)$, and $g^{\prime} \mid \sigma-1$.

For any integer $s$ let $M(s)$ denote the multiplicative group of the reduced residues modulo $s$. If $(x, s)=1$, let $\{x\}_{\text {o }}$ denote the cyclic subgroup of $M(s)$ generated by $x$. For the integer $n$ above and any $x$ with $(x, n)=1$, we let $\{x\}_{n}=\{x\}$. Let $t$ be the order of $\{r\}$ and for any $s \mid n$, let $t_{s}$ be the order of $\{r\}_{s}$.

Now let $s$ and $s^{\prime}$ be positive integers dividing $n$. We define an equiv-

Received by the editors August 14, 1968. 
alence relation, $s \sim s^{\prime}$, with respect to $h$ and $r$. For any prime $p$ dividing $n$ let $p^{x} \| n$, i.e. $p^{x} \mid n$ and $p^{x+1} \nmid n, p^{\pi}\left\|h, p^{z}\right\| s$, and $p^{z^{\prime}} \| s^{\prime}$. Then $s \sim s^{\prime}$ if the following conditions are satisfied:

(i) If $p \neq 2$, then either $x \geqq \pi+z$ and $x \geqq \pi+z^{\prime}$ or $z=z^{\prime}$.

(ii) If $p=2$ and $4 \mid r-1$, then the conditions are as in (i) above.

(iii) If $p=2,4 \mid r+1$, and $x<\pi+z$, then $z=z^{\prime}$.

(iv) If $p=2,4 \mid r+1,2^{x-\pi} \| r+1$, and $x \geqq \pi+z$, then $z \leqq 1$ and $z^{\prime} \leqq 1$.

(v) If $p=2,4\left|r+1,2^{x-\pi+1}\right| r+1$, and $x \geqq \pi+z$, then $z=z^{\prime}$.

Note that in (iii), (iv), and (v) we only need the cases where $z \leqq 1$ and $z^{\prime} \leqq 1$.

Now we put the integers $n, h, g$, and $g^{\prime}$ in the canonical forms and define some other integers.

Let $n=\prod p^{x}, \quad h=\prod p^{\pi}, g=\prod p^{z}, g^{\prime}=\prod p^{z^{\prime}}, \nu=\prod_{\left.p\right|_{r-1}} p^{x}$, and $\mu=n / \nu$. If a discussion is about a prime $p$, then the exponents $x$, $\pi, \cdots$, will have the above meanings for this prime $p$. However, whenever confusion is likely, we add a subscript, e.g., $x=x_{p}$.

Let $\eta=\nu / 2$ when $n \equiv h g \equiv 0(\bmod 8), g-2 \equiv r+1 \equiv 0(\bmod 4)$, and $2 \pi\} t_{\mu}$ and let $\eta=\nu$ otherwise. For $p \mid \nu$, let $p^{u} \| t_{\nu}$ and $p^{v} \| t_{\mu}$. Finally let

$$
\begin{aligned}
\theta_{1} & =2 n\left(r^{t_{\mu}}-1\right) /\left(2^{x}, n\right), \quad \theta_{2}=\left(2, t_{\nu}, t_{\mu}, g\right)(r-1), \\
\theta & =\left(\left[\left[\theta_{1}, h g\right] / g h, \theta_{2}, k\right], n\right)=\prod p^{w}, \quad \text { and } \lambda=\theta / \mu .
\end{aligned}
$$

Now we give the main result of this paper.

Theorem. Let $G$ and $H$ be given as above. Then $G$ and $H$ are isomorphic if and only if $g \sim g^{\prime},\{r\}_{\eta}=\{\sigma\}_{\eta}$, and $\{r\}_{\theta}=\{\sigma\}_{\theta}$.

We give the rule to determine all the nonisomorphic extensions of a cyclic group of order $n$ by a cyclic group of order $h$. Let $L$ be the set we get by taking a generator of every cyclic subgroup of $M(n, h)$ $=\left\{r \in M(n) \mid r^{h} \equiv 1(\bmod n)\right\}$. If $4 \nmid n$ or $2 \nmid h$ let $L^{\prime}=L$. If $4 \mid n$ and $2 \mid h$ let $L^{\prime}=\{r \in L|4| r-1\}$ and $L^{\prime \prime}=\{r \in L|4| r+1\}$.

First, take the set $L^{\prime}$ and consider the equivalence relation between the divisors of $n$ where $r \in L^{\prime}$. [Note we may assume $4 \mid r-1$ for all $r \in L^{\prime}$ when $L=L^{\prime}$. Hence the equivalence relation is defined by (i) and (ii) only.] Let $S$ be the set of all smallest integers from each equivalence class. [The smallest integer of an equivalence class divides every other integer from the class and the reason for picking this integer follows from the remark in \$4.] For $g \in S$ let $L^{\prime}(g)$ $=\left\{r \in L^{\prime}|g| r-1\right\}$. Pick an $r \in L^{\prime}(g)$ and define integers $\eta$ and $\theta$ as above and eliminate all $\sigma^{\prime}$ s in $L^{\prime}(g)$ satisfying the conditions of the theorem. Do this until every $r \in L^{\prime}(g)$ is either picked or eliminated for all $g \in S$.

Second, take the set $L^{\prime \prime}$, i.e. when $4 \mid n$ and $2 \mid h$. Consider the 
equivalence relation on the divisors of $n$ given by (i) and

(ii') If $p=2$ then either $z=z^{\prime}=0$ or $z=z^{\prime}=1$.

Let $S^{\prime}$ be the set of all smallest integers from each equivalence class. For $g \in S^{\prime}$ let $L^{\prime \prime}(g)=\left\{r \in L^{\prime \prime}|g| r-1\right\}$. If $g$ is odd, then pick any $r \in L^{\prime \prime}(g)$. If $g$ is even, then pick $r \in L^{\prime \prime}(g)$ such that $2^{x-\pi+1} \mid r+1$ when $x \geqq \pi+1$ and any $r \in L^{\prime \prime}(g)$ when $x<\pi+1$. Now proceed as above for all $g \in S^{\prime}$.

The required nonisomorphic groups have the defining relations as of $G$ above where $g$ runs over all elements of $S$ and, if $4 \mid n$ and $2 \mid h$, over all elements of $S^{\prime}$ with the integers $r$ selected as above.

3. Preliminary lemmas. The following lemmas are used extensively in the proof of the theorem. Let $p$ be a prime and $r$ an integer.

LEMMA 1. Let $p \neq 2$ and $p^{x} \| r-1$ with $x \geqq 1$, or let $p=2$ and either $2^{x} \| r-1$ or $2^{x} \| r+1$ with $x \geqq 2$. Then $p^{x+y} \| r^{p^{y}}-1$ where $y>0$.

LemMa 2. Let $R=\{r\}$ be a cyclic subgroup of $M\left(p^{y}\right)$ of order $p^{x}$. If $p \neq 2$, then $r=1+p^{y-x}$ with $0 \leqq x \leqq y-1$. If $p=2$, then $r=1+2^{y-x}$ or $-1+2^{y-x}$ for $2 \leqq x \leqq y-2$ and $r=1+2^{y-1},-1+2^{y-1}$, or -1 for $x=1$.

Lemma 3. Assume the hypothesis of Lemma 1.

(i) If $p^{w} \mid r^{2}-1$ where $w>x$, then $p^{w-x} \mid z$.

(ii) If $p^{x} \| r-1, p \nmid z$, and $w>0$, then $p^{w} \| 1+r+\cdots+r^{z p^{w}-1}$.

(iii) If $2^{x} \| r+1,2 \nmid z$, and $w>0$, then $2^{w+x-1} \| 1+r+\cdots+r^{22^{w}-1}$.

Lemma 4. Let $(n, m)=1,\{r\}_{n}$ of order $t$, and $\{\sigma\}_{m}$ of order $\tau$. Then $\left\{\sigma^{\prime}\right\}_{n m}$ is of order $[t, \tau]$ where $\sigma^{\prime} \equiv r(\bmod n)$ and $\sigma^{\prime} \equiv \sigma(\bmod m)$.

LeMma 5. In Lemma 4 replace $r$ and $\sigma$ by $r^{\alpha}$ and $r^{\beta}$ respectively, where $(\alpha, t)=(\beta, \tau)=1$. Then $\left\{\sigma^{\prime}\right\}_{n m}=\{r\}_{n m}$ if and only if $(t, \tau) \mid \alpha-\beta$.

To prove Lemma 1 , let $r=1+z p^{x}$ or $-1+z 2^{x}$, as suitable where $p \nmid z$. The result follows by considering the binomial expansion of $r^{p^{\nu}}$ for the given cases. The proofs of Lemmas 2 and 3 follow. For the proofs of Lemmas 4 and 5 use the Chinese remainder theorem.

From this point on we fix our notations as given in $\$ 2$. Let $G^{\prime}$ and $Z$ be the commutator group and the center of $G$ respectively. Then $G^{\prime}=\left\{a^{r-1}\right\}$ and is of order $n^{\prime}=n /(r-1, n)$ and $Z=\left\{a^{n^{\prime}}, b^{t}\right\}$ and is of order $h(r-1, n) / t$.

Lemma 6. If $G \cong H$ then $g \sim g^{\prime},(r-1, n)=(\sigma-1, n)$, and $\{r\}$ and $\{\sigma\}$ are of equal order in $M(n)$.

Proof. We only need to prove $g \sim g^{\prime}$ since the others follow from the note above. Assume $g \times g^{\prime}$. We take the different cases and show that $G$ and $H$ are not isomorphic. For some prime $p$ let $x<\pi+z^{\prime}$ and $z<z^{\prime}$. Then $H$ has an element, namely $d$, whose order is divisible by 
$p^{\pi+z^{\prime}}$. Let $m=n h g / p^{x}$ if $\pi+z>x$ and $m=n h g / p^{\pi+z}$ if $x \geqq \pi+x$. Then for any integer $\beta$ we have $r^{\beta m} \equiv 1(\bmod n)$. From the factorization of the last congruence and Lemma 3 we have

$$
1+r^{\beta}+\cdots+r^{\beta(m-1)} \equiv 0 \quad(\bmod n) .
$$

Also we have $\left(a^{\alpha} b^{\beta}\right)^{m}=1$ for all integers $\alpha$ and $\beta$. Hence there exists no element of $G$ whose order is divisible by $p^{\pi+z^{\prime}}$ and therefore $G \neq H$.

Now assume $p=2, x \geqq \pi+1,2^{x-\pi+1} \mid r+1, \pi>0$, and $0=z^{\prime} \neq z=1$. From Lemma 3 we have $2^{x} \mid 1+r+\cdots+r^{2 \pi-1}$. Hence the order of $a^{\alpha} b^{\beta}$ is exactly divisible by $2^{\pi+1}$ if $\beta$ is odd. Assume it is possible to set $G=\left\{a^{\alpha} b^{\beta}, a^{\delta} b^{5}\right\}$ such that the order of $\left\{a^{\alpha} b^{\beta}\right\} \cap\left\{a^{\delta} b^{5}\right\}$ is odd and $\left\{a^{\alpha} b^{\beta}\right\}$ is normal of order $n$. Then $2 \mid \zeta$ and hence $\beta$ is odd. Hence we only need to consider the case $x=\pi+1$. It could be shown that the normality of $\left\{a^{\alpha} b^{\beta}\right\}$ implies that the 2-Sylow subgroup of $G$ is quaternion of order 8 , a contradiction. This completes the proof of the lemma.

LEMмA 7. If $\{r\}=\{\sigma\}$ and $g=g^{\prime}$, then $G \cong H$.

Proof. Let $\sigma \equiv r^{s}(\bmod n)$ where $(s, t)=1$. Let $\beta$ be a solution of $\beta \equiv s(\bmod t)$ and $\beta \equiv 1\left(\bmod m^{\prime}\right)$ where $m^{\prime}$ is the product of the distinct primes $p, p \mid h g$ and $p \nmid t$. Let $\alpha$ be the solution of $\alpha \equiv \beta(\bmod g)$ and $\alpha \equiv 1\left(\bmod n^{\prime}\right)$ where $n^{\prime}$ is the product of the distinct primes $p, p \mid n$ and $p \nmid g$. Then the map $\psi: H \rightarrow G$ where $\psi(c)=a^{\alpha}$ and $\psi(d)=b^{\beta}$ is an isomorphism.

4. Metacyclic $p$-groups and a general remark. Let $p$ be a prime and let $n=p^{x}, h=p^{\pi}$, and $g=g^{\prime}=p^{z}$, i.e. $G$ and $H$ are metacyclic $p$-groups. For $p=2, x \geqq 3, \pi \geqq 2, z=1$, and $4 \mid r+1$ let $\eta=n / 2=2^{x-1}$, otherwise let $\eta=n=p^{x}$.

LEMMA 8. Let $G$ and $H$ be p-groups as above. Then $G \cong H$ if and only if $\{r\}_{\eta}=\{\sigma\}_{\eta}$.

Proof. If $p \neq 2$, the result is immediate from Lemmas 6,7 , and 2 . The same is true when $p=2$ and $4 \mid r-1$.

Now assume $\eta=n / 2$, with the above conditions. If $\{r\}_{\eta}=\{\sigma\}_{\eta}$, then either $\{r\}=\{\sigma\}$ or $r+1 \equiv \sigma+1+2^{x-1} \equiv 0\left(\bmod 2^{x}\right)$. In the first case $G \cong H$ from Lemma 7 and in the latter case $\psi: H \rightarrow G$, given by $\psi(c)=a b^{2 \pi-1}$ and $\psi(d)=b$, is an isomorphism.

For the necessity, from Lemmas 6 and 2, we only need to consider the case where $r+1 \equiv \sigma+1+2^{x-1} \equiv 0\left(\bmod 2^{x}\right)$. Since $g \mid r-1, z=0$ or 1 . For $x=2, G$ and $H$ are not isomorphic. Hence let $x \geqq 3$. Assume $G$ and $H$ are isomorphic and the isomorphism $\psi: H \rightarrow G$ is given by $\psi(c)=a^{\alpha} b^{\beta}$ and $\psi(d)=a^{\delta} b^{5}$. These give two congruences (see $\S 5$ ). The first is $\beta\left(-2+2^{x-1}\right) \equiv 0\left(\bmod 2^{\pi}\right)$. The second, for the case where $\beta$ is even, is 


$$
\alpha(-1)^{\zeta} \equiv \alpha\left(-1+2^{x-1}\right)+2^{x-\pi-z} \beta\left(-2+2^{x-1}\right) \quad\left(\bmod 2^{x}\right) .
$$

Assume $z=0$. If $\pi>1$, then $\beta$ is even, $\zeta$ is odd and hence $\alpha$ is even and $a^{\alpha} b^{\beta}$ is of order at most $2^{x-1}$. If $\pi=1$ and $\beta=0$, then as above $\alpha$ is even and $a^{\alpha} b^{\beta}$ is at most of order $2^{x-1}$. If $\pi=1$ and $\beta=1$, then $\left(a^{\alpha} b^{\beta}\right)^{2}$ $=1$. Hence in these cases $G \not \neq H$.

Assume $z=1$. The only case left is when $\pi=1$. But in this case $G$ is a generalized quaternion group and has only one cyclic subgroup of order 2 , namely $\left\{b^{2}\right\}$. The subgroups $\left\{d^{2}\right\}$ and $\{c d\}$ are distinct subgroups of order 2 of $H$. Hence $G \not \equiv H$, and the proof is complete.

REMARK. We make a note on the general case. Using the integer $g$ we define an integer $g_{0}$ as the product of all $p^{z}$ where $p \mid g$ and either $x<\pi+z$ or for $p=2$ and $4\left|r+1,2^{x-\pi+1}\right| r+1$. Then $g_{0}$ is the smallest integer of the equivalence class containing $g$. Let $\zeta=1+r+\cdots$ $+r^{h-1}$. Let $\beta$ be an integer such that $\beta \equiv p^{x-\pi-z}\left(\bmod p^{x}\right)$ if $p \mid g$ and $x \geqq \pi+z$ for $p \neq 2$ or for $p=2$ with $4 \mid r-1$. For $2 \mid g$ and $4 \mid r+1$ let $\beta \equiv 1\left(\bmod 2^{x}\right)$. Otherwise let $\beta \equiv 0\left(\bmod p^{x}\right)$. Then from Lemma 3 , for $p \mid g, x \geqq \pi+z$, where $p \neq 2$ or $p=2$ and $4 \mid r-1$, we have $p^{x-z} \| \beta \zeta$. For $2 \mid g$ and $4 \mid r+1$ we have $2^{\delta+\pi-1} \| \beta \zeta$ where $2^{\delta} \| r+1$. Note that for $p=2$ and $4 \mid r+1$ we have $z=1$ or 0 and $\delta \geqq x-\pi$.

Let $\alpha$ be an integer such that $\alpha(\beta \zeta / k) \equiv-1\left(\bmod p^{z}\right)$ whenever $p^{x-z} \| \beta \zeta$ and $\alpha \equiv 0\left(\bmod p^{z}\right)$ otherwise. [The first case also includes the case where $p=2, z=1,4 \mid r+1$, and $\delta=x-\pi$.] It could be easily shown that $a^{\alpha \beta} b$ is of order $h g_{0}$. Hence $G$ is isomorphic to a group $G_{0}$ whose defining relations are given by those of $G$ with $g_{0}$ exchanged for $g$ and $k_{0}=n / g_{0}$ exchanged for $k$.

Assume $G \cong H$ and $4 \mid r+1$ with $4 \mid n$ and $2 \mid h$. Consider the 2-Sylow subgroups $G(2)$ and $H(2)$ of $G$ and $H$ respectively. Then $G(2) \cong H(2)$. From Lemma 7 the defining relations of $G(2)$ and $H(2)$ can be taken as those of $G$ and $H$ where $n, h, g$, and $g^{\prime}$ are changed to $2^{x}, 2^{x}, 2^{z}$, and $2^{z^{\prime}}$ respectively. From Lemma 6 the highest power of 2 dividing $r+1$ is the same as that dividing $\sigma+1$ except possibly when $r+1$ $\equiv \sigma+1+2^{x-1} \equiv 0\left(\bmod 2^{x}\right)$. Assume $r+1 \equiv \sigma+1+2^{x-1} \equiv 0\left(\bmod 2^{x}\right)$. If $z=z^{\prime}$, then Lemma 8 implies that $\pi \geqq 2$. If $1=z \neq z^{\prime}=0$ and $\pi=1$, then $G(2) \neq H(2)$ since $G(2)$ is the quaternion group. If $0=z \neq z^{\prime}=1$ and $\pi=1$, then $z^{\prime}=1$ may be changed to $z^{\prime}=0$ (see proof of Lemma 8) and hence $G(2) \neq H(2)$. Hence we have $\pi \geqq 2$ in case $r+1 \equiv \sigma+1+2^{x-1}$ $\equiv 0\left(\bmod 2^{x}\right)$, and therefore $2^{x-x+1}$ divides both $r+1$ and $\sigma+1$.

The above discussion shows that the equivalence relation on the divisors of $n$ will be the same if $\sigma$ is exchanged for $r$ provided that $G \neq ⿻$ H. Hence $g_{0}$ and $k_{0}$, as defined above, may be exchanged for $g^{\prime}$ and $k^{\prime}$ in the defining relation of $H$. This implies that we only need to prove the theorem for the case where $g^{\prime}=g$. 
5. Proof of the necessity. In the next two sections assume the notations of $\$ 2$ and let $g=g^{\prime}$. For convenience we let $m=h g=\prod p^{y}$ where $\pi=y-z$.

Let $p$ be a prime dividing $\nu$. Then using the Sylow theorem and Lemma 7, the defining relations of any $p$-Sylow subgroup, $G(p)$, of $G$ is given by

$$
A^{p^{x}}=B^{p^{y}}=1, \quad A^{p^{x-z}}=B^{p^{y-z}}, \quad B A=A^{r} B .
$$

Here $r$ is of order $p^{u_{p}}$ in $M\left(p^{x}\right)$. Similarly the $p$-Sylow subgroup, $H(p)$ $=\{C, D\}$, of $H$, is given by exchanging $C$ for $A, D$ for $B$, and $\sigma$ for $r$. Let $\eta_{p}$ be defined for the prime $p$ as done in $\$ 4$. The isomorphism of $H(p)$ and $G(p)$, by Lemma 8 , gives us $\sigma \equiv r s_{p}\left(\bmod \eta_{p}\right)$ where $\left(s_{p}, p\right)$ $=1$. Using Lemma 5 we have $\sigma \equiv r_{p}^{s}\left(\bmod \eta^{\prime}\right)$ where $\eta^{\prime}$ is the product of all $\eta_{p}, p \mid \nu$ and $s$ is an integer. Note that $\eta^{\prime}=\eta$ for all cases except when $4 \mid r+1$ and $2^{y-1} \mid t_{\mu}$, in which case $\eta^{\prime}=\eta / 2$.

Assume the isomorphism, $\psi: H \rightarrow G$, is given by $\psi(c)=a^{\alpha} b^{\beta}$ and $\psi(d)=a^{\delta} b^{5}$. Then from $\psi(d c)=\psi\left(c^{\sigma} d\right)$ we have

$$
\begin{gathered}
\beta(\sigma-1) \equiv 0 \quad(\bmod h) \\
\alpha r^{5} \equiv \alpha\left(1+r^{\beta}+\cdots+r^{\beta(\sigma-1)}\right)+\delta\left(r^{\beta \sigma}-1\right) \\
+\beta k(\sigma-1) / h \quad(\bmod n) .
\end{gathered}
$$

(a) $r^{\beta} \equiv 1(\bmod \mu)$. Let $p \mid \mu$, then $p \nmid \sigma-1$ and from (i), $p^{y} \mid \beta$. Assume $r^{\beta} \not \equiv 1\left(\bmod p^{x}\right)$, then since $r^{h} \equiv 1\left(\bmod p^{x}\right)$, from Lemma $1, p \nmid r^{\beta}-1$. Choose an integer $f$ such that $p \nmid f$ and $q^{\tau_{q}}\left|f, q^{y_{q}}\right| f, q \neq p$. Then $r^{f \beta} \equiv 1$ $\left(\bmod q^{x_{q}}\right)$ for every $q \mid n$. Using this and Lemma 3 , (ii) and (iii), it can be shown that $\left(a^{\alpha} b^{\beta}\right)^{f}=1$, a contradiction. Hence $r^{\beta} \equiv 1(\bmod \mu)$ and $t_{\mu} \mid \beta$.

(b) $(\alpha, \mu)=1$ and $\sigma \equiv r^{\zeta}(\bmod \mu)$. Let $p \mid \mu$ and assume $p \mid \alpha$. Choose $f$ such that $n \nmid f, n / p \mid f$ and $m \mid f \beta$. As above we have $\left(a^{\alpha} b^{\beta}\right)^{f}=1$, a contradiction. Hence $(\alpha, \mu)=1$. The second result follows since $\mu \mid \beta k(\sigma-1) / h$. And since we have an isomorphism $\psi^{\prime}: G \rightarrow H$, we also have $\left(\zeta, t_{\mu}\right)=1$.

(c) $\left(\alpha, t_{p}, t_{\mu}\right)=1$. We have

$$
a=\left(a^{\alpha} b^{\beta}\right)^{e}\left(a^{\delta} b^{5}\right)^{f}=a^{i} b^{\beta e+\zeta f}
$$

where $\beta e+\zeta f \equiv 0(\bmod h), i \equiv 1(\bmod k)$, and

$$
i=\alpha\left(1+r^{\beta}+\cdots+r^{\beta(e-1)}\right)+\delta r^{\beta e}\left(1+r^{5}+\cdots+r^{5(f-1)}\right) .
$$

If $p \mid t_{\nu}$ and $p \mid t_{\mu}$, then $p \mid \beta$ and $p \mid k$. From the above $p \mid f$, and hence from Lemma $3, p$ divides the last sum in the expression for $i$. Since $i \equiv 1(\bmod k)$, it follows that $p \nmid \alpha$ or $\left(\alpha, t_{\nu}, t_{\mu}\right)=1$.

(d) $\theta \mid \beta k(\sigma-1) / h$. Clearly $k \mid \beta k(\sigma-1) / h=n^{\prime}$. To prove $\left(\theta_{2}, n\right)$ 
divide $n^{\prime}$ we only need to show that $\beta k / h$ is an even integer when $t_{\nu}, t_{\mu}$, and $g$ are all even. Now $\beta k / h$ is an integer since $\left(a^{\alpha} b^{\beta}\right)^{k}=\left(a^{\delta} b^{\zeta}\right)^{h}$ $=a^{f k}$ where $(f, g)=1$ and

$$
f=\alpha\left(1+r^{\beta}+\cdots+r^{\beta(k-1)}\right) / k+\beta k / h .
$$

Assume $t_{\nu}, t_{\mu}$, and $g$ are all even. Then $2 \nmid \alpha, 2|\beta, 4| r^{\beta}-1$, and from Lemma 3, the coefficient of $\alpha$ is odd. Hence $\beta k / h$ is even since $f$ is odd.

To show that $\left(\left[\theta_{1}, m\right] / m, n\right) \mid n^{\prime}$, we only need to consider primes $p$ dividing $\nu$. Considering the highest power of $p$ dividing $\left(\left[\theta_{1}, m\right] / m, n\right)$, the result can be proved easily. Note that for $p=2$, the cases $4 \mid r-1$ and $4 \mid r+1$ should be taken separately.

Now we complete the proof of the necessity. First note that if $2^{y-1} \mid t_{\mu}$ then $2^{x} \mid \theta$ and, from congruence (ii), $\sigma \equiv r^{s}\left(\bmod 2^{x}\right)$, and hence $\sigma \equiv r^{s}(\bmod \eta)$. This implies $\sigma \equiv r^{s}(\bmod \lambda)$. For $p \mid \nu$, replacing $p^{w}$ for $n$ in congruence (ii) and using the above we have

$$
\alpha r^{\zeta} \equiv \alpha \sigma+f p^{v+w-u^{\prime}} \quad\left(\bmod p^{w}\right)
$$

where $p^{u^{\prime}} \| t_{\lambda}$. [Note that the cases $p=2,4 \mid r-1$ and $p=2,4 \mid r+1$ should be taken separately.] Replacing $\sigma$ by $r^{8}$ in this congruence, it follows that $\left(t_{\lambda}, t_{\mu}\right) \mid s-\zeta$. Since $\theta=\lambda \mu$, using (b) above and Lemma 5 we have $\{\sigma\}_{\theta}=\{r\}_{\theta}$. It is already shown above that $\{\sigma\}_{\eta}=\{r\}_{\eta}$, which proves the necessity.

6. Proof of the sufficiency. The conditions of the theorem mean that there exist integers $e$ and $f$ such that $\sigma \equiv r^{e}(\bmod \eta)$ and $\sigma \equiv r^{f}$ $(\bmod \theta)$ where $\left(e, t_{\eta}\right)=\left(f, t_{\theta}\right)=1$. If $\eta=\nu / 2$ then either $\sigma \equiv r^{e}\left(\bmod 2^{x}\right)$ or $r+1 \equiv \sigma+1+2^{x-1} \equiv 0\left(\bmod 2^{x}\right)$. For the defining relations of $H$ we assume $\{c\} \cap\{d\}$ is of order $g$ instead of merely $c^{k}=d^{h}$.

We find integers $\alpha, \beta$, and $\zeta$ such that the map $\psi: H \rightarrow G$, given by $\psi(c)=a^{\alpha} b^{\beta}$ and $\psi(d)=b^{5}$, is an isomorphism. We fix $e$ and $f$ above and define an integer $\theta^{\prime}$.

For $p \mid \nu$ let $p^{s^{\prime}} \| r^{e}-r^{f}$. Let $p^{s} \| \theta^{\prime}, p \mid n$, where $s$ is given as follows:

For $p \neq 2$ or $p=2$ and $\sigma \equiv r^{e}\left(\bmod 2^{x}\right)$, let $s=x$ if $p \mid r-1, v=0, z=0$, $s^{\prime} \geqq x$, or $s^{\prime} \geqq x+v-u$, otherwise let $s=s^{\prime}$. For $p=2$ and $r+1$ $\equiv \sigma+1+2^{x-1} \equiv 0\left(\bmod 2^{x}\right)$, let $s=x-1$. Define $\lambda^{\prime}=\theta^{\prime} / \mu$, then $\sigma$ $\equiv r^{e}\left(\bmod \lambda^{\prime}\right)$. Applying Lemmas 3 and 5 it could be shown that there exists $f^{\prime}$ such that $\sigma \equiv f^{\prime}\left(\bmod \theta^{\prime}\right)$.

Now we set the congruences (a), (b), and (c), that define integers $\zeta$, $\beta$, and $\alpha$ respectively.

(a) $\zeta \equiv f^{\prime}\left(\bmod t_{\theta^{\prime}}\right)$ and $\zeta \equiv 1\left(\bmod e^{\prime}\right)$ where $e^{\prime}$ is the product of distinct primes $p$ with $p \mid m$ and $p \nmid t_{\theta^{\prime}}$. 
(b) $\beta \equiv 0\left(\bmod p^{y}\right)$ if $s=x, \beta \equiv p^{s-2 x+y+u}\left(\bmod p^{y}\right)$ if $s<x$ and either $p \neq 2$ or $p=2$ and $4 \mid r-1$, and $\beta \equiv 2^{s-x+y+1}\left(\bmod 2^{y}\right)$ if $4 \mid r+1$.

(c) $\alpha \equiv 1\left(\bmod p^{x}\right)$ if $s=x$. If $s<x$, then

$$
\alpha\left[r^{r}-\left(1+r^{\beta}+\cdots+r^{\beta(\sigma-1)}\right)\right] \equiv \beta k(\sigma-1) / h \quad\left(\bmod p^{x}\right) .
$$

Now consider the prime $p \mid \nu$ and assume $x<s$. For $p$ odd, from Lemma $1, p^{x-u} \| r-1$, and from the definition of $\theta$ we have $s \geqq w$ $\geqq x+(x-u+v)-y$ or $p^{v} \mid y$. For $p=2$, two cases occur and again $2^{v} \mid y$. Again, using the definition of $\theta, s \geqq w \geqq x-z$, and hence $h \mid \beta(\sigma-1)$.

Now for $s<x, p \neq 2$, we have $p^{s} \| \beta k(\sigma-1) / h$. Also $r^{\beta} \equiv 1\left(\bmod p^{s+1}\right)$, for otherwise $s=x$. Hence the coefficient of $\alpha$ in the second congruence of (c) is $r^{\xi}-r^{e}\left(\bmod p^{s+1}\right)$. From the definition of $\zeta, p^{s} \| r^{\xi}-r^{e}$. For $p=2$, two cases occur and $2^{s} \| r^{5}-r^{e}$. Hence $\alpha$ exists.

From the above definitions we have $b^{\zeta}\left(a^{\alpha} b^{\beta}\right)=\left(a^{\alpha} b^{\beta}\right)^{\sigma} b^{5}$. Again using prime divisors $p$ of $\nu$ as above, we have $h \mid y k$. Using Lemma 3, it follows that $\left(a^{\alpha} b^{\beta}\right)^{k}$ is a power of $a^{k}$, say $a^{f k}$. Here $f$ is given as in (d) of $\S 5$. Note that $(\alpha, n)=1$. If $p \mid g$ then $p \mid r-1$. From Lemma 3 and the definition of $\beta$ the prime $p$ does not divide the first expression in the formula of $f$. If $p=2,2 \mid g$, then from the definition of $\beta$ we have $2 \mid \beta k / h$ in all cases and hence $2 \nmid f$. For $p \neq 2, s=x$, we have $p \mid \beta k / h$ and $p \nmid f$. Now let $p \neq 2$ and $s<x$ and assume $p \mid f$. Then the expression above gives $\alpha=-\beta k / h+f^{\prime} p$ for some integer $f^{\prime}$. Putting this in the second congruence of $(\mathrm{c})$ we have, since $r^{\beta} \equiv 1\left(\bmod p^{s+1}\right)$,

$$
-\beta k\left(r^{5}-\sigma\right) / h \equiv \beta k(\sigma-1) / h \quad\left(\bmod p^{s+1}\right)
$$

or $p^{s+1}$ divides $-\beta k\left(r^{\xi}-1\right) / h$. But $p^{x-u} \| r^{\xi}-1$ and $s \geqq x-u$, and hence $p \mid \beta k / h$. This implies that $p \nmid f$, a contradiction. Therefore $(f, g)=1$ and $\left(a^{\alpha} b^{\beta}\right)^{k}$ is of order $g$ and hence $H$ and $G$ are isomorphic. This proves the sufficiency and completes the proof of the theorem.

\section{BIBLIOGRAPHY}

1. Y. Gol'fand, On an isomorphism between extensions of groups, Dokl. Akad. Nauk SSSR 60 (1948), 1123-1125.

2. G. Szekeres, Metabelian groups with two generators, Proc. Internat. Conf. Theory of Groups, Austral. Nat. Univ. Canberra, August 1965, pp. 323-346; Gordon and Breach, New York, 1967.

University of Nebraska at OMaha 